\title{
A systematic review of the evidence for deprescribing interventions among older people living with frailty
}

Kinda Ibrahim ${ }^{1,2^{*}}$, Natalie J. Cox ${ }^{1,3}$, Jennifer M. Stevenson ${ }^{4,5}$, Stephen Lim ${ }^{1,2}$, Simon D. S. Fraser ${ }^{2,6}$ and Helen C. Roberts ${ }^{1,2,3}$

\begin{abstract}
Background: Older people living with frailty are often exposed to polypharmacy and potential harm from medications. Targeted deprescribing in this population represents an important component of optimizing medication. This systematic review aims to summarise the current evidence for deprescribing among older people living with frailty.

Methods: The literature was searched using Medline, Embase, CINAHL, Psyclnfo, Web of Science, and the Cochrane library up to May 2020. Interventional studies with any design or setting were included if they reported deprescribing interventions among people aged $65+$ who live with frailty identified using reliable measures. The primary outcome was safety of deprescribing; whereas secondary outcomes included clinical outcomes, medication-related outcomes, feasibility, acceptability and cost-related outcomes. Narrative synthesis was used to summarise findings and study quality was assessed using Joanna Briggs Institute checklists.

Results: Two thousand three hundred twenty-two articles were identified and six (two randomised controlled trials) were included with 657 participants in total (mean age range 79-87 years). Studies were heterogeneous in their designs, settings and outcomes. Deprescribing interventions were pharmacist-led $(n=3)$ or multidisciplinary teamled $(n=3)$. Frailty was identified using several measures and deprescribing was implemented using either explicit or implicit tools or both. Three studies reported safety outcomes and showed no significant changes in adverse events, hospitalisation or mortality rates. Three studies reported positive impact on clinical outcomes including depression, mental health status, function and frailty; with mixed findings on falls and cognition; and no significant impact on quality of life. All studies described medication-related outcomes and reported a reduction in potentially inappropriate medications and total number of medications per-patient. Feasibility of deprescribing was reported in four studies which showed that 72-91\% of recommendations made were implemented. Two studies evaluated and reported the acceptability of their interventions and further two described cost saving.

(Continued on next page)
\end{abstract}

\footnotetext{
* Correspondence: k.ibrahim@soton.ac.uk

${ }^{1}$ Academic Geriatric Medicine, Faculty of Medicine, University of Southampton, Southampton, UK

${ }^{2}$ NIHR Applied Research Collaboration Wessex, Southampton, UK

Full list of author information is available at the end of the article
}

C C The Author(s). 2021 Open Access This article is licensed under a Creative Commons Attribution 4.0 International License, which permits use, sharing, adaptation, distribution and reproduction in any medium or format, as long as you give appropriate credit to the original author(s) and the source, provide a link to the Creative Commons licence, and indicate if changes were made. The images or other third party material in this article are included in the article's Creative Commons licence, unless indicated otherwise in a credit line to the material. If material is not included in the article's Creative Commons licence and your intended use is not permitted by statutory regulation or exceeds the permitted use, you will need to obtain permission directly from the copyright holder. To view a copy of this licence, visit http://creativecommons.org/licenses/by/4.0/. The Creative Commons Public Domain Dedication waiver (http://creativecommons.org/publicdomain/zero/1.0/) applies to the data made available in this article, unless otherwise stated in a credit line to the data. 
(Continued from previous page)

Conclusion: There is a paucity of research about the impact of deprescribing in older people living with frailty. However, included studies suggest that deprescribing could be safe, feasible, well tolerated and can lead to important benefits. Research should now focus on understanding the impact of deprescribing on frailty status in high risk populations.

Trial registration: The review was registered on the international prospective register of systematic reviews (PROSPERO) ID number: CRD42019153367.

Keywords: Frailty, Deprescribing, Medication review, Polypharmacy, Inappropriate medications

\section{Background}

One-third of people aged over 65 years live with multimorbidity and take five or more regular medicines (polypharmacy), increasing to $50 \%$ in over 85 year olds $[1,2]$. Polypharmacy in older people is associated with increased risk of serious adverse events, falls, cognitive impairment, functional decline, hospitalisation, length of stay and death, [3-5]. Such harms are amplified in older people living with frailty, a complex geriatric syndrome resulting in decreased physiological reserve [6]. In frail older people the harms might outweigh benefits for some medications e.g. intensive blood glucose control in type 2 diabetes, or the known time to benefit exceeds projected life expectancy e.g. statins [7, 8]. Additionally, the goals of drug treatment in older people living with frailty may change compared with older people in general, shifting the focus from reducing the risk of disease and prolonging life to reducing the burden of treatment and maintaining quality of life [9]. The bi-directional relationship between polypharmacy and frailty has been reported. Drugs and frailty might interact through network of connections, including physiological changes, multiple pathologies and chronic diseases, life expectancy and functional or cognitive status [10-14]. Frailty may influence factors such as drug pharmacokinetics and pharmacodynamics, toxicity, and therapeutic efficacy. In turn, these factors may be involved in the development of frailty [15].

The cure for polypharmacy appears simple and involves deprescribing - the process of tapering /dose reduction, stopping, or switching drugs, with the goal of managing polypharmacy and improving outcomes [16]. There has been considerable research conducted on deprescribing since the term was first used in 2003 [17], and more recently there has been a focus on deprescribing for those living with frailty. Several tools have been developed to assist physicians with deprescribing decisions such as STOPPFrail [18, 19]. However, investigation of the impact of deprescribing on those living with frailty has been limited to date.

Several systematic reviews have synthesised the evidence on outcomes of deprescribing interventions among older people in general $[20,21]$, or defined by setting including care homes [22, 23], primary care and community [24, 25] and hospitals [26]. These reviews reported that deprescribing is feasible, well tolerated, safe, and generally effective in reducing the number of inappropriate prescriptions. However, these reviews either did not include frail older people or frailty was poorly defined in their included studies, for example based on age or setting such as being in a care home with definition subject to international variability [27]. There is an increasing awareness that identifying frail older people or those at risk of frailty using reliable tools should be part of routine clinical practice, to guide appropriate interventions to improve clinical outcomes [28]. The dynamic nature of frailty highlights a potential for preventive and restorative interventions to maintain the capacity for self-care and to prevent disabilities, falls, functional decline, institutionalisation, hospitalisation and death [29]. For example, it could be crucial to identify older people living with frailty and polypharmacy as priority patients for a medication review and deprescribing intervention, which could potentially reduce medication-related harm and improve patients' outcomes. Using objective, reliable measures to assess frailty in the context of research studies on deprescribing is also important to assess whether study results can be extrapolated to patients with similar scores, or to measure whether frailty status affects response to deprescribing interventions and vice versa.

Therefore, the aim of this systematic review was to explore the safety and impact of deprescribing among older people living with frailty identified by reliable measures.

\section{Methods}

\section{Data sources and searches}

The search strategy was developed with a senior librarian and used the following databases: Medline, Embase, CINA HL, PsycInfo, Web of science, and the Cochrane library from database conception until January 2020. Keywords such as deprescribing, deprescribe*, polypharmacy, inappropriate prescribing were used (see Appendix). Reference lists of retrieved articles were searched for additional relevant studies. The search was re-run in May 2020 but 
no further eligible papers were retrieved. The review was carried out using the methods recommended by the Preferred Reporting Items for Systematic reviews and MetaAnalyses (PRISMA) statement [30] and was registered on the international prospective register of systematic reviews (PROSPERO) ID number: CRD42019153367.

\section{Study inclusion \\ Type of studies}

We anticipated a small number of studies to explore deprescribing in frail older people. Only interventional studies with any design, setting or language were included (Table 1).

\section{Type of participants}

We included interventions that targeted an older population with a median age of 65 years and over, who are identified to be frail using reliable measures including but not limited to Fried Frailty Phenotype, FRAIL scale, PRISMA-7, electronic-Frailty Index, Edmonton Frail Scale Gérontopôle Frailty Screening Tool and Clinical Frailty Scale. To be included, studies had to have at least $50 \%$ of their study population identified as frail.

\section{Type of interventions}

Studies involving deprescribing as the only intervention or as part of medication review intervention where deprescribing accounts for at least 50\% of the total recommendations were included. Studies where deprescribing formed part of a multi-dimensional intervention (such as in combination with nutritional and physical activity components) were excluded as it is difficult to ascertain which component of the intervention was responsible for the reported outcomes.

\section{Type of outcomes}

The primary outcome we chose was safety of deprescribing. We defined safety in terms of reported adverse events, hospital admission and/or all-cause mortality.

Secondary outcomes included clinical outcomes (such as frailty status, function, falls, cognition, depression, quality of life), medication-related outcomes (such as changes in number of medications and Potentially Inappropriate Medications PIMs), feasibility of deprescribing (defined by the number of patients/proportion who successfully stopped medications), its acceptability by patients or healthcare practitioners, and cost-related outcomes.

\section{Study selection}

Two authors (KI \& NC) independently screened the title and abstracts of identified articles using the Rayyan electronic platform to identify studies that met the inclusion criteria [31]. Following each stage, any disagreement was resolved by discussion.

\section{Quality assessment}

Study quality was assessed separately by two authors (SL \& SF) using the standardized Joanna Briggs Institute checklists for each study type, with total scores of 13 for randomized controlled trials (RCTs) and 9 for nonrandomised experimental studies. Final scoring was agreed by discussion. A score $\geq 7 / 13$ for RCTs and 5/9 for non-randomised experimental trials were considered to represent good quality.

\section{Data abstraction and synthesis}

Due to heterogeneity of study designs and outcome measures, quantitative synthesis (meta-analysis) was not possible and narrative synthesis of the findings was conducted following the Synthesis Without Meta-analysis (SWiM) guideline [32]. Data from included studies were extracted independently by two authors (KI \& JS) into a pre-defined template for conceptualisation and construction of the literature review (Table 2). Data abstracted included: year of publication, country, setting, number and age of participants, description of the deprescribing intervention and any comparator, types and classes of medications most frequently deprescribed, frailty measures used, deprescribing tools and outcomes of deprescribing. Studies were grouped according to intervention type (pharmacist-led or multidisciplinary team-led) due to the heterogeneity of study designs and outcomes. Outcome data were summarised for each study and compared.

Table 1 PICO statement for study inclusion

\begin{tabular}{|c|c|}
\hline Population & Older people (mean age $65+$ ) who live with frailty measured objectively by any reliable tool. \\
\hline Intervention & $\begin{array}{l}\text { Studies at any setting and any language that included deprescribing medication review (including tapering/dose reduction, stopping } \\
\text { or switching drugs). Deprescribing as the only intervention or part of medication review where deprescribing accounts for at least } \\
50 \% \text { of changes. }\end{array}$ \\
\hline Comparator & Any, or no, comparator considered \\
\hline Outcomes & $\begin{array}{l}\text { Primary outcome: safety of deprescribing } \\
\text { Secondary outcomes: clinical outcomes, medication-related outcomes, feasibility of deprescribing, acceptability and cost-related } \\
\text { outcomes. }\end{array}$ \\
\hline
\end{tabular}




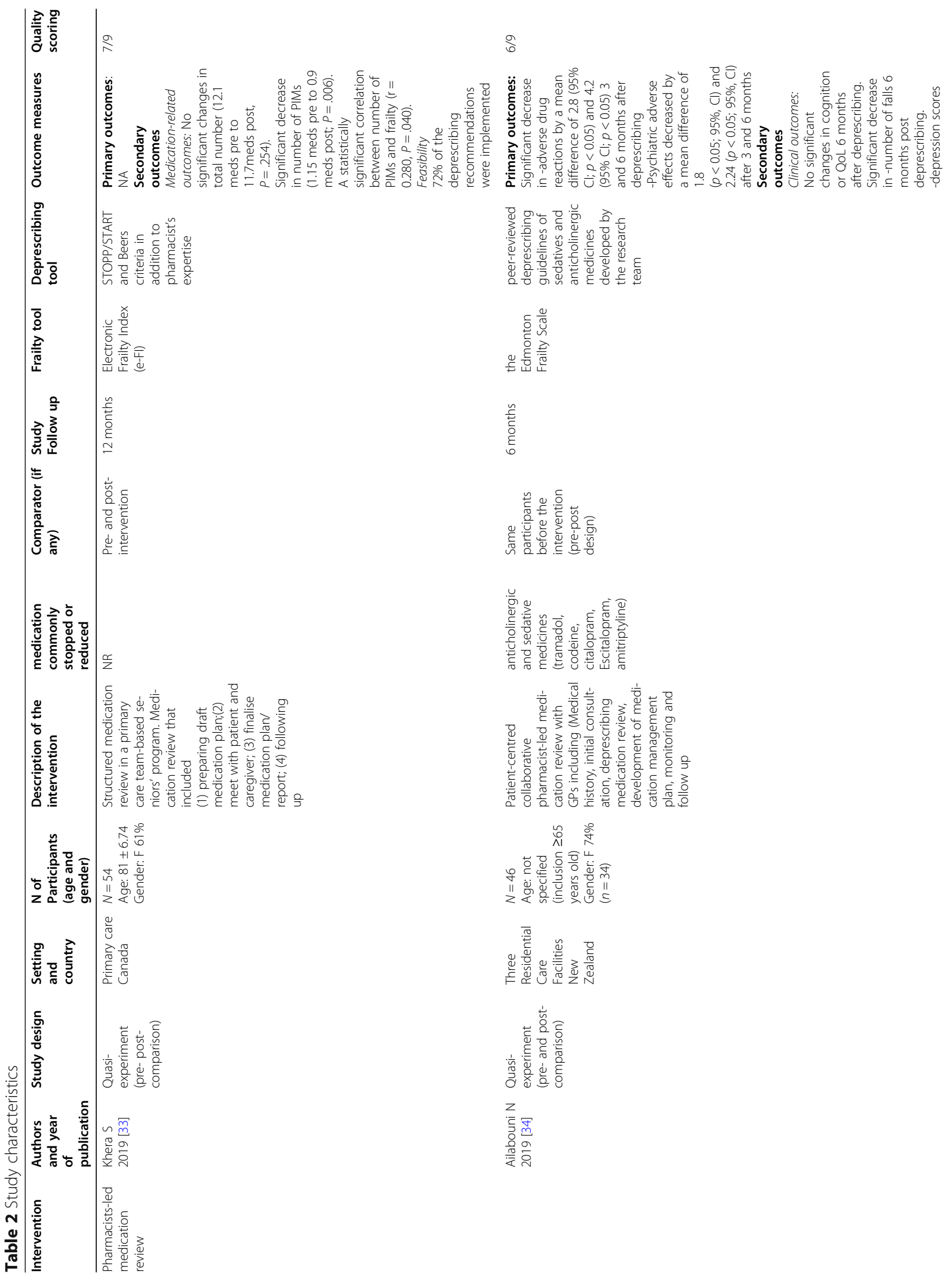




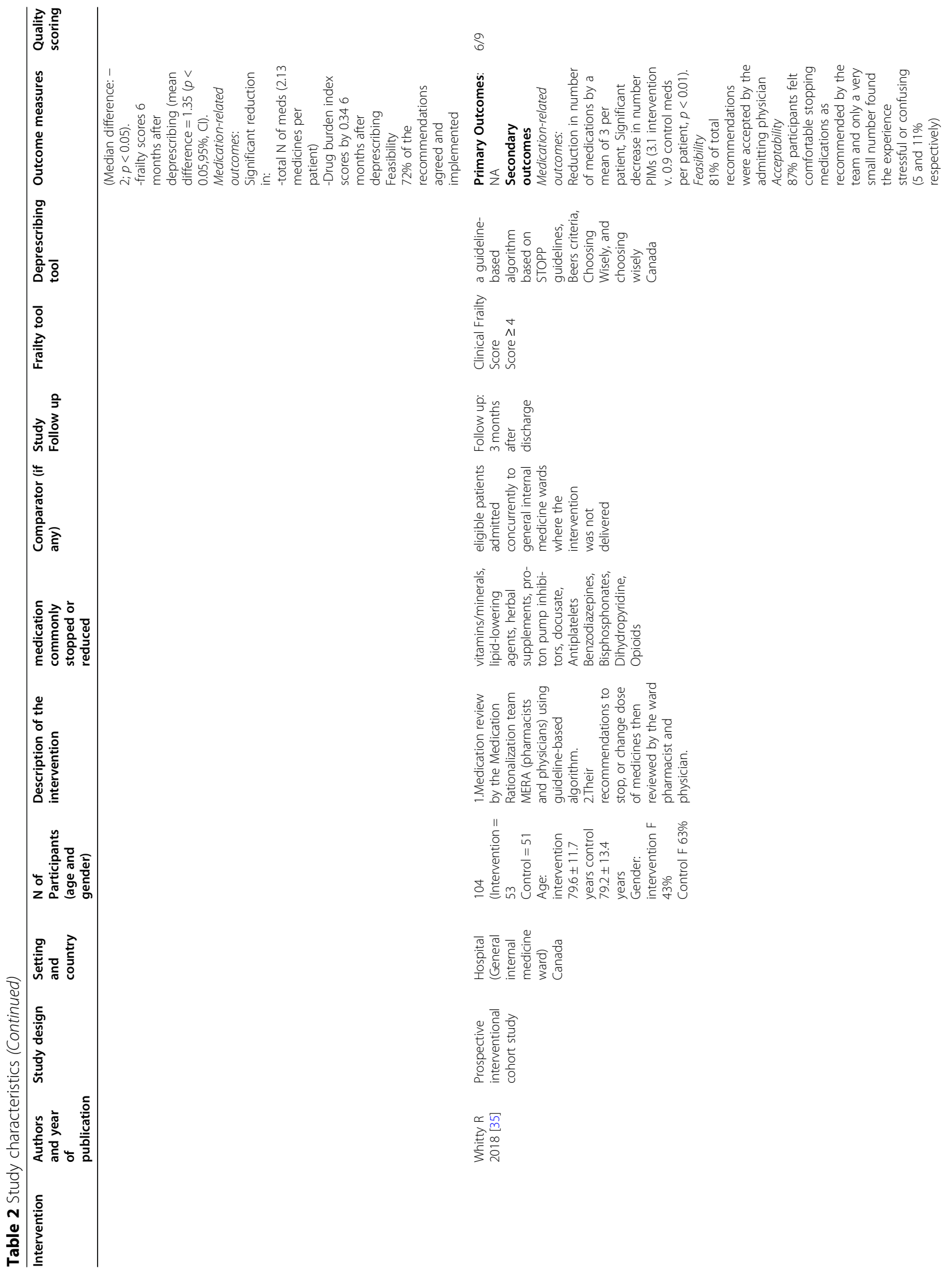




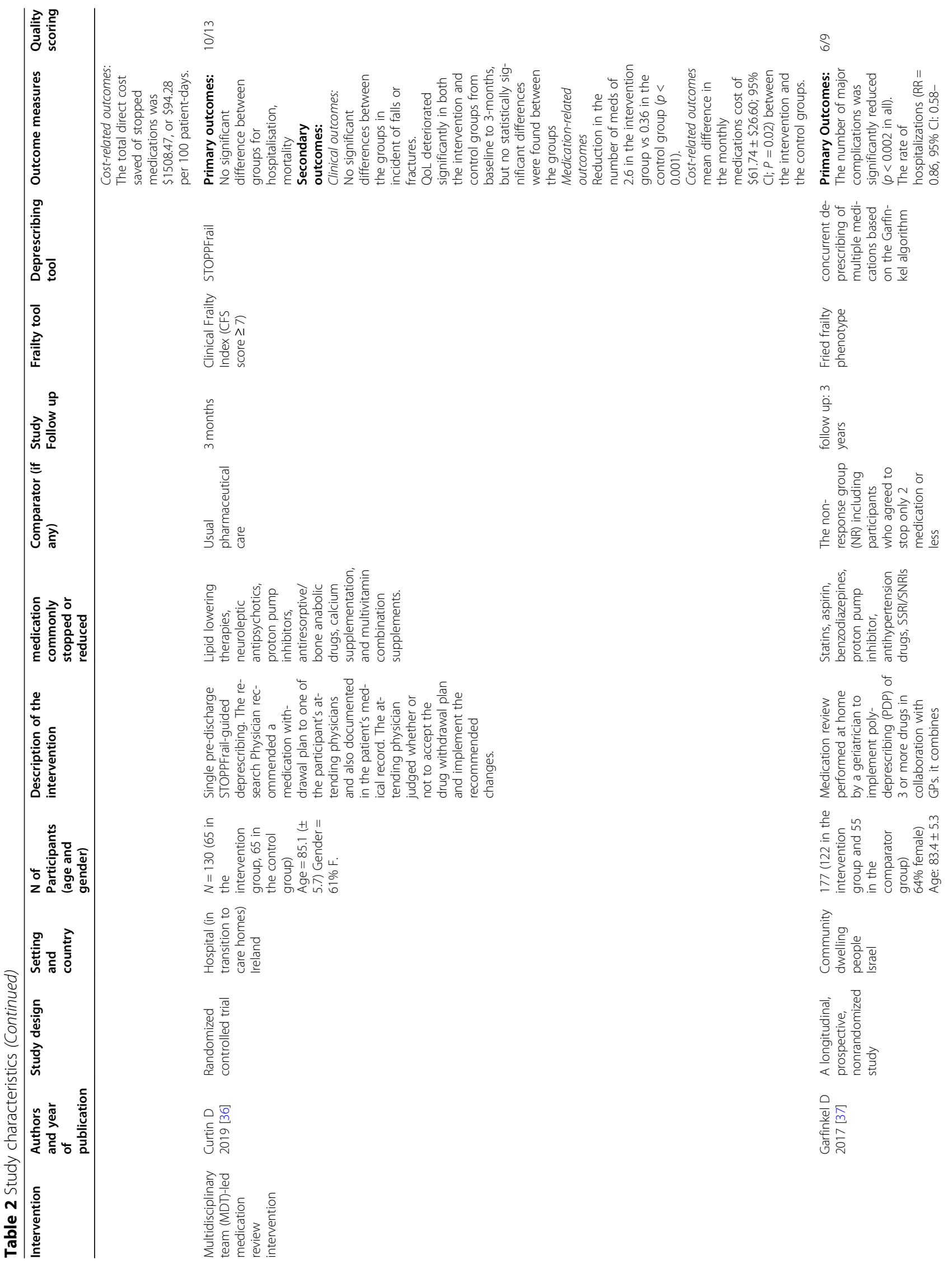




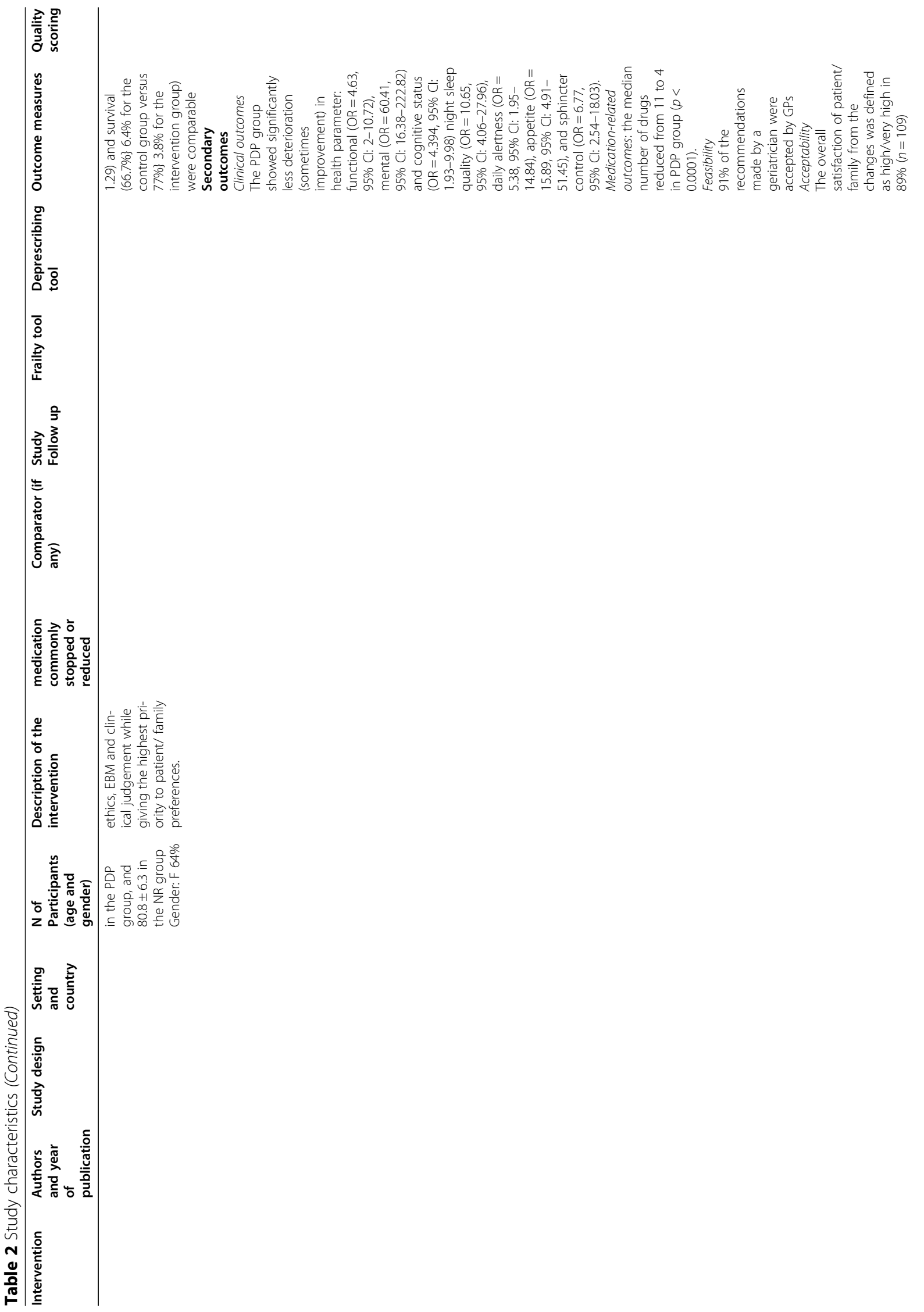




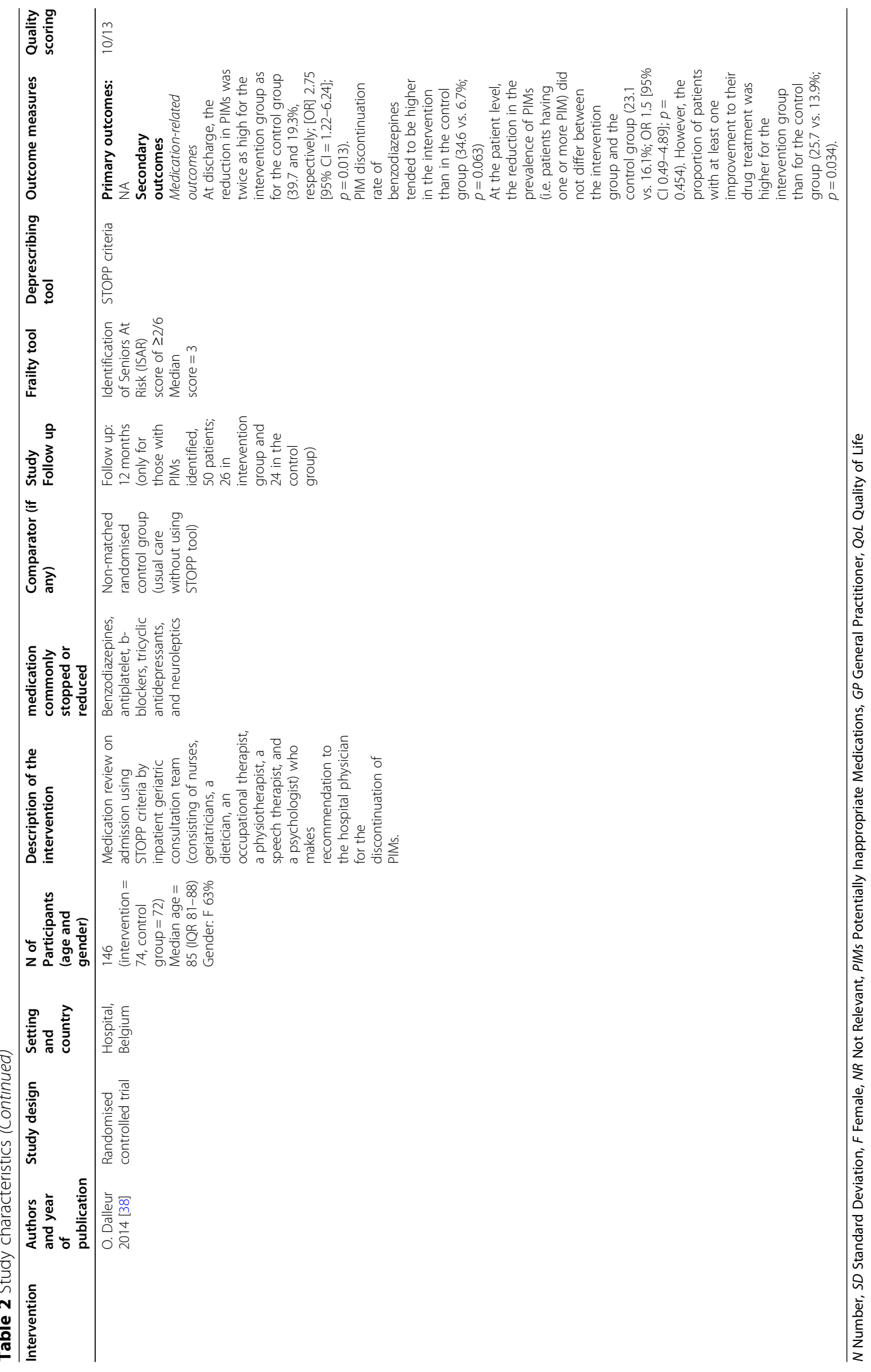




\section{Results}

Two thousand three hundred twenty-two articles were identified, and 57 articles were selected for full text assessment from which six journal articles were included in this review (Fig. 1). Six conference abstracts were excluded due to limited information available and poor quality of reporting. No articles in any language other than English were identified. The quality of the full text articles included was good: $\geq 6 / 9$ for the four nonrandomised experimental studies and 10/13 for the two randomised-controlled trials RCTs.

\section{Participants characteristics}

The total number of participants in all included studies was 657 , while individual sample sizes ranging from 46 to 177 and mean participant age range from 79 to 85 years. The Canadian Clinical Frailty Score (CFS) was the most commonly used frailty measure $(n=2)[35,36]$, with others including: the Edmonton Frailty Scale [34], Identification of Seniors At Risk (ISAR) [38], the
Electronic Frailty Index (e-FI) [33], and Fried Frailty Phenotype [37].

\section{Study characteristics and deprescribing interventions}

This review includes two RCTs, two pre- and post- comparison studies and two prospective interventional cohort studies (Table 2). Studies were conducted in Ireland, Belgium, New Zealand, Canada, and Israel. Study settings included: hospital (3), care home (1), primary care (1), community (1). All included articles were published between 2014 and 2019.

Several tools and algorithms were used to guide deprescribing. Two studies used explicit criteria (lists of drug names targeted); one used the STOPP criteria [38], and one used the STOPPFrail tool [36]. Two studies utilised implicit criteria (lists of evaluative questions or a process) developed by the research teams themselves including the Garfinkel algorithm for concurrent deprescribing of multiple medications [37], and guidelines for targeted deprescribing of anticholinergic and sedative medicines [34]. The remaining two studies used a

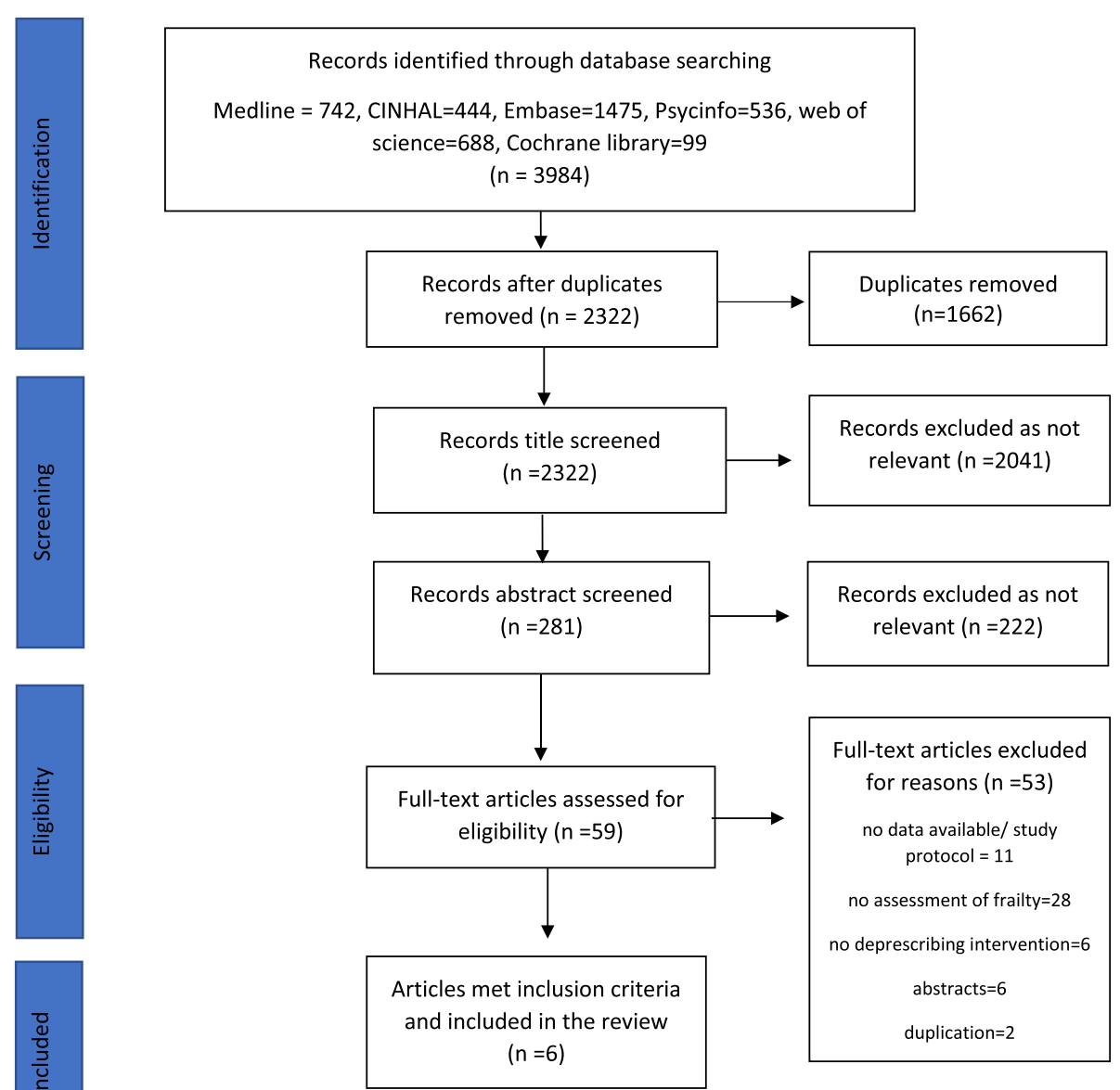

Fig. 1 PRISMA Flow Diagram of identification of articles 
combination of both explicit and implicit criteria; one used STOPP and Beer's alongside pharmacist's judgment [33], and the second used an algorithm based on STOPP guidelines, Beers criteria, Choose Wisely and Choose Wisely Canada [35]. The medications most frequently deprescribed across the studies regardless of the setting were: benzodiazepines, antidepressants, neuroleptics, opiates, lipid-lowering agents (statins), vitamin and nutritional supplements, proton pump inhibitors, and cardiovascular drugs (aspirin, antiplatelets, b-blockers, digoxin).

Due to heterogeneity of the outcome measures and study designs, studies were grouped according to interventions: pharmacist-led deprescribing interventions $(n=3)$ and multidisciplinary team-led intervention $(n=3)$.

\section{Pharmacist-led deprescribing}

Three of the six studies described pharmacist-led deprescribing interventions: one across three care home facilities [34], one in primary care (across six practices) [33] and one in hospital [35]. The three studies were nonrandomised experimental studies with a good quality score, $>6 / 9$. Follow up periods were 3 months (after discharge from hospital), 6 months (in primary care) and twelve months (in care home).

The care home and primary care studies used a person-centred, collaborative, pharmacist-led deprescribing medication review with the General Practitioner (GP) [33, 34]. Both interventions involved a detailed medication review process that engaged patients and their relatives in decisions about medication discontinuation; both drafted and shared a medication plan and followed patients for close monitoring. While the hospital study was a prospective interventional cohort study that employed medication reviews by a Medication Rationalization (MERA) team, involving physicians and led by pharmacists, for 53 frail inpatients (CFS $\geq 4$ ). These were compared with 51 patients in the control arm who did not receive the MERA review [35].

In the care home study, the pharmacist used implicit guidelines of sedatives and anticholinergic medicines developed by the research team among 46 residents identified to be frail using Edmonton Frailty Scale [34]. Whereas in the two studies in primary and secondary care, pharmacists used the STOPP and Beers criteria to identify prescribing problems among 54 community dwelling older people with some degree of frailty using the electronic frailty index (e-FI) [33] and 53 frail inpatients using the Clinical Frailty Score (CFS $\geq 4$ ) [35].

\section{Multidisciplinary team (MDT)-led deprescribing}

Three studies implemented multidisciplinary team-led deprescribing focused medication review: two were
RCTs in hospital settings (good quality score of 10/13) and one was a longitudinal prospective interventional study in community (good quality, 6/9). Follow up periods were three and twelve months in hospital studies and 3 years in the community study.

One RCT of 146 patients (74 in the intervention group vs 72 in the control group) living with frailty (ISAR $\geq 2$ / 6) implemented medication review using STOPP criteria on admission by an inpatient geriatric team consisting of nurses, geriatricians, a dietician, an occupational therapist, a physiotherapist, a speech therapist, and a psychologist [38]. Another RCT of 130 inpatients (65 in the intervention group vs 65 in the control group) living with frailty (CFS score $\geq 7$ ) used a STOPPFrail-guided deprescribing intervention by a research physician predischarge to a care home [36]. A longitudinal prospective nonrandomized study among 177 community dwelling older people living with frailty (median Fried Frailty Phenotype $=3$ ) employed concurrent deprescribing of multiple medications based on the Garfinkel algorithm by a geriatric consultant in collaboration with GPs [37]. The intervention group included patients who had 3 or more medications deprescribed (Poly-De-Prescribing PDP group $n=122$ ), while the control group included participants who agreed to stop only 2 medications or less $(n=55)$.

\section{Outcomes of deprescribing}

The outcomes reported in the included studies are presented in Table 2; safety of deprescribing in three studies; clinical outcomes $(n=3)$; medication-related outcomes $(n=6)$; feasibility of deprescribing $(n=4)$; acceptability $(n=2)$ and cost-related outcomes $(n=2)$.

\section{Primary outcome}

Safety of deprescribing Three studies reported the safety of deprescribing and its impact on adverse events [34, 36, 37]. Potential adverse drug reactions using the UKU-SERS score, in a pharmacist-led deprescribing intervention among 46 care home residents, decreased by a mean difference of $2.8(95 \% \mathrm{CI} ; p<0.05)$ after 3 months and $4.2(95 \% \mathrm{CI} ; p<0.05)$ after 6 months of deprescribing of sedative and antipsychotic medications [34]. In addition, adverse effects of psychotropic medications decreased significantly by a mean difference of 1.8 (95\%, CI; $p<0.05) 3$ months after deprescribing, and by a mean difference of $2.24(95 \%, C I ; p<0.05)$ after 6 months of deprescribing. One RCT in hospital reported that $88 \%$ of deprescribing recommendations based on STOPPFrail were accepted and implemented and no adverse events were reported of MDT-led deprescribing during 3 months follow-up [36]. 
Two MDT-led deprescribing studies in hospital and community showed no significant differences in unplanned hospitalisation and mortality [36, 37]. The RCT in hospital showed no statistically significant differences between the intervention and control groups for 3 months unscheduled hospital presentations (0.14 intervention vs 0.08 control, $95 \% \mathrm{CI}, P=0.27$ ) and mortality ( 0.18 vs $0.28,95 \%$ CI; $P=0.22$ ) [36] . Similarly a longitudinal cohort study in community reported that the incidence of hospitalisations per patient per year (0.39 intervention vs 1.02 comparator, $p=0.1006$ ) and survival (77\% intervention vs $67 \%$ comparator group, $p=0.026$ ) was comparable between the groups after 3 years [37]. This study also reported that the incidence of significant complications per patient/year was significantly reduced in the PDP group [0.22 intervention vs 1.72 comparator group, $p=0.0047$ ] [37].

\section{Secondary outcomes}

Clinical outcomes Frailty and function: Two studies reported the outcomes of deprescribing on frailty and function [34, 37]. Pharmacist-led deprescribing sedatives and anticholinergic medicines among 46 care home residents showed a significant decrease in frailty scoring (mean difference of $1.35,95 \% \mathrm{CI}, P<0.05$ ) using the Edmonton Frailty scale, after 6 months of deprescribing. Another pharmacist-led deprescribing study did not report whether deprescribing led to changes in frailty status but reported a positive and statistically significant correlation between number of PIMs (using STOPP and Beers criteria) and frailty using e-FI $(r=0.280, P=.040)$ [33]. The impact of deprescribing on functional status defined using a 5 point scale $(1=$ independent, $2=$ frail, $3=$ mild disability, $4=$ disability, $5=$ severe disability) was examined in another MDT-led deprescribing study [37]. Patients in the poly-deprescribing group had less functional deterioration compared to the comparator group [37] (69.1\%) vs $42(34.4 \%), P<0.001)$.

Falls: The impact on falls was mixed on reports from two studies [34, 36]. Significant decreases in falls rate, defined as the number of falls in the past 90 days, was reported after pharmacist-led deprescribing psychotropic medicines among care home residents [34]. But on the other hand, falls risk (determined using an in-house falls risk assessment tool utilised by most residential care facilities in New Zealand) remained the same 6 months after deprescribing in the same study. Another MDT-led deprescribing RCT study using STOPPFrail at hospital discharge reported no significant difference in incidence of falls $(0.27$ vs $0.30,95 \% \mathrm{CI}, P=0.75)$ and non-vertebral fractures $(0.02$ vs $0.09,95 \%, P=0.18)$, among patients who moved to nursing home after 3 months of deprescribing [36].
Cognition, depression and mental health status: two studies reported outcomes on cognition, depression and mental status [34, 37]. No change in cognition using the interRAI cognitive performance scale was observed after 3 and 6 months of pharmacist-led deprescribing of antipsychotic medications among care home residents (mean difference of $0, p=0.26$ ). However, significant improvement of depression scores using the geriatric depression scale (GDS) (mean difference of $-2, p<0.05$ ) were seen after 6 months [34]. Another MDT-led deprescribing study reported that patients in the polydeprescribing (PDP) group had improvement in mental status using the Mini Mental State Examination (MMSE) test (3 comparator vs 63 intervention, $p<0.0001$ ) and cognitive status (0 comparator vs 7 intervention, $P=$ 0.0004) [37]. These improvements occurred within 3 months after deprescribing in $83 \%$ and persisted for $\geqslant 2$ years in $68 \%$.

Quality of life (QoL): Two studies assessed QoL of participants and showed no significant differences after implementing deprescribing interventions $[34,36]$. QoL among care home residents was assessed using EQ-5D$3 \mathrm{~L}$ and showed no significant difference pre and 6 months post deprescribing [34]. QoL in one RCT in hospital using QUALIDEM or ICECAP-O scores showed deterioration in both the intervention and the control group from baseline to 3 months follow-up, but no statistically significant differences were found in the mean changes between groups [36].

Medication-related outcomes All six studies reported medication-related outcomes [33-38]. Four studies reported a significant reduction in the number of medications taken by patients living with frailty after implementing deprescribing, ranging from a mean of $2-$ 3 medicines stopped per patient [34-36] across the different settings to unsurprisingly 7 medications per patient when poly-deprescribing of three or more drugs was implemented in people's home [37]. Two studies also reported significant decreases of potentially inappropriate medications associated with deprescribing interventions $[33,38]$; for example the deprescribing interventions in two hospital studies reported a mean decrease in number of PIMs of $2.2(p<0.01)$ in a pharmacist-led study and reduction in PIMs was twice as high for the intervention group compared to the control group in a MDT-led deprescribing intervention. One care home study reported a significant decrease in the drug burden index (by 0.34) 6 months after deprescribing in care home residents with a pharmacist-led deprescribing intervention [34].

Feasibility of deprescribing Four studies reported the feasibility of deprescribing among older people with 
frailty [34-37]. They displayed that $72-91 \%$ of the suggestions to deprescribe medications made by either pharmacists or the MDT were accepted and implemented across the different settings [34-37]. For example, in care homes, forty-five PIMs were identified and suggested to be stopped by pharmacists, of which $82 \%$ were agreed upon by the residents' GP and $96 \%$ were agreed upon by the resident or their relatives/family resulting in the implementation of $72 \%$ of the recommendations [34]. Similarly in two hospital studies, 72 and $81 \%$ of the recommendations made were accepted and implemented by the admitting physician and then patients $[35,36]$. In one community study, $91 \%$ of the recommendations made by a geriatrician were accepted by GPs [37].

Deprescribing was also reported to be well tolerated as most medications stopped were not restarted. For example, in care homes, medicines were re-prescribed by the GP in only five instances (15\%); stopping medication was not completed in 13 residents $(28 \%)$ due to mood changes, increased pain levels or overall health deterioration [34]. Similarly in hospital, of the 162 medications that were stopped only $40(25 \%)$ were restarted during hospital admission or at time of discharge and $81 \%$ of medications stopped during hospitalisation remained discontinued after 3 months [35]. Another RCT study among hospitalised older patients discharged to care homes showed that only three medications stopped at discharge by the MDT were restarted [36].

Acceptability of deprescribing Two studies evaluated the acceptability of their deprescribing interventions and showed that patients and healthcare professionals were happy to stop unnecessary medication [35, 37]. For example, following a pharmacist-led intervention $87 \%$ participants felt comfortable stopping medications as recommended by the team and only a small number found the experience stressful or confusing (5 and 11\% respectively) [35]. In the poly-deprescribing intervention in community, the overall satisfaction of patient/ family from the changes was defined as high/very high in $89 \%$ [37].

Cost-related outcomes Two studies reported the cost implications of deprescribing [35, 36]. A pharmacist-led intervention reported a total saving of $\$ 1508.47$ or $\$ 94.28$ per 100 patient-days when STOPP criteria were implemented in hospital [35]. Use of STOPPFrail by an MDT at discharge from hospital also led to a mean change in monthly medication cost of $-\$ 74.97$ compared to $-\$ 13.22$ in the control group (mean difference $\$ 61.74$; $95 \% \mathrm{CI} ; P=.02)[36]$.

\section{Discussion}

This review expands on prior literature reviews by synthesising studies on medication deprescribing that specifically addressed older people living with frailty, as they are more vulnerable to the adverse effects of medicines compared to older people in general. Only six studies (two were RCTs) with overall good quality that reported the outcomes of deprescribing interventions among older people, with reliably identified frailty, were found. The outcomes of deprescribing in older people living with frailty were similar to those reported in older people in general in terms of feasibility, acceptability and safety, as mortality and hospitalisation rates did not increase after stopping medications. Deprescribing interventions led to a significant reduction in the number of medications and PIMs with potential cost saving. Included studies also suggest some evidence of potential improvements in function, frailty status, mental health and depression scores. Outcomes did not differ when the intervention was led by a pharmacist or MDT including mainly medical practitioners and whether explicit or implicit criteria were used. But the heterogeneous study designs limit our ability to make firm conclusions regarding this matter.

Deprescribing medications has raised some ethical dilemmas and fear of negative outcomes has been reported by prescribers as a barrier to deprescribing [39]. Among older people with identified frailty, there is some evidence from the included studies in this review that deprescribing is safe, as it did not adversely change hospitalisation and mortality rates. A number of systematic reviews have investigated the impact of deprescribing on mortality among general population of older people; one reported that deprescribing reduced mortality in nonrandomized studies but no changes were observed in RCTs [40]; other reviews suggested a reduction in allcause mortality with deprescribing interventions in nursing home residents $[22,23]$. We reported some evidence that deprescribing is feasible and well tolerated by older people living with frailty and is acceptable by healthcare professionals and patients, which is in agreement with existing studies in older people in general [41, 42]. In our review we identified that $72-91 \%$ of recommendations made were implemented and very few patients (25\%) restarted their medications. A recent review of 26 papers reported the proportion of patients who successfully stopped their medication varied from 20 to $100 \%$ and in 19 studies the proportion was $>50 \%$ [24]. The feasibility and safety of deprescribing should encourage clinicians to regularly discuss the decision to continue or deprescribe chronic medications with their patients living with frailty, following a patient-centred, structured deprescribing process with planning, tapering and close monitoring during, and after medication withdrawal. 
Few studies in the review reported clinical outcomes such as frailty, falls, cognition and depression; with more focus placed on the success of the interventions in reducing number of medications and especially inappropriate ones. This focus on process and lack of clinical outcome data with inconsistency in outcome measurement have also been highlighted as limitations in deprescribing studies to date. A 2017 review of deprescribing interventional studies among older people in general reported the outcome measures most commonly used were number of medications or PIMs stopped, healthcare use, and adverse events [43]. Patient-reported outcomes, geriatric syndromes (e.g. falls, fractures, gait speed, depression and delirium) or cost evaluations were infrequently reported, and frailty was not used as either inclusion criteria or an outcome measure. There is no consensus among researchers and clinicians on appropriate outcomes of deprescribing and more research is needed in this area. Frailty should be considered as an outcome in deprescribing interventions in older people and the focus should be placed on understanding the impact of deprescribing on frailty trajectory.

The strong relationship between polypharmacy and frailty and the potential to reverse frailty status [44, 45], makes it important to understand the impact of deprescribing on frailty. Only one study included in our review examined the impact of deprescribing on frailty status among 46 care home residents using the Edmonton frailty tool and reported positive results [34]. The Edmonton frailty tool consists of 9 domains including number of medications [46]. It is unclear from the study which domains were influenced by the deprescribing intervention or to what extent the improvement could simply reflect a decrease in the number of medications used. Another included study reported that frailty and PIMs were significantly correlated but did not report the impact of deprescribing on frailty status. There is a lack of research on the impact of stopping medications on frailty status but some current registered clinical trials propose to measure this relationship [47, 48]. It is also important to understand the mechanism by which deprescribing might influence frailty via functional or cognitive changes or through other possible mechanisms.

No effect of deprescribing on the quality of life among older people with frailty was reported in our review. These findings are consistent with literature published in older people in general $[20,21,49,50]$. Possible explanations for this might be that the impact of deprescribing on QoL may depend on the specific combination of medication(s), patient population and patients' preferences, clinical setting, timing of QoL measurement or the QoL measurement tools used. We found a positive impact of deprescribing sedative and psychotic medications using a specific algorithm on rate of falls among older care home residents living with frailty, but no similar impact was obtained when the STOPPFrail tool was used among hospitalised patients discharged to care homes. This might be explained by the fact that the deprescribing algorithm focused on sedative and psychotic medications resulting in a higher proportion of anticholinergic medications being stopped compared to a tool with a broader remit like STOPPFrail. The inconsistency in reported findings regarding the relationship between falls and deprescribing is clear in the literature. For example, a recent review published in 2017 reported that falls-risk drug withdrawal strategies did not significantly change the rate of falls, number of people who fell or rate of fall-related injuries over a 6 to 12 months follow-up period in five included papers [51]. However, another review suggested that deprescribing interventions could significantly reduce the number of people who fall in care homes by $24 \%$ [22]. They related this to the significant reduction in number of residents on PIMs by $60 \%$ such as anticholinergics, which have been consistently associated with cognitive impairment and falling in older people. As we mentioned above, the impact of deprescribing on falls could be mediated by the deprescribing tools used and further research should explore this relationship.

The intervention process, who led deprescribing or the deprescribing tools used, appeared to have no differing effects in reducing unnecessary medications in our review. But the heterogeneity in study designs and the small number of included studies limit our ability to conclude whether one approach is more or less effective than another. Other reviews suggested that pharmacist-led deprescribing intervention in older people in general were more effective in reducing unnecessary medications compared to interdisciplinary team interventions $[52,53]$. The concurrent use of both explicit lists of potentially inappropriate medications and systematic appraisal of every medication taken was suggested to help improve complex regimes [54]. Deprescribing techniques may be guided by the clinical situation. Stopping medicines one at a time might be most appropriate for managing people whose health status is stable in out-patient settings, whereas 'concurrent deprescribing' of multiple medications may be more appropriate for inpatients where it is easier to monitor for withdrawal effects [54]. It is also recommended to use deprescribing as a 'drug holiday trial' as sometimes drugs will need to be restarted when symptoms recur or withdrawal effects are experienced, which necessitates monitoring and follow up [54].

This review is the first to summarise the evidence and impact of deprescribing among older people identified as living with frailty. Most published reviews focused on the general population of older people or in a specific setting. 
With the increasing awareness of the importance of identifying frailty using reliable measures to allow implementation of effective interventions, this review expands our knowledge of the evidence of deprescribing among this population who are more vulnerable to harm from medications. However, there were several limitations in our review. The inclusion criteria required a reliable and valid measure of frailty. This is important to allow extrapolation of the study results to patients with similar scores, or to measure whether frailty status affects response to deprescribing interventions. However, we may have excluded articles assessing frail older people but which utilised less specific methods of assessing frailty or those that assumed frailty depending on age or setting such as studies in care homes. Multicomponent interventions including deprescribing or medication review where deprescribing accounted for less than half of the recommendations were excluded, as our aim was to understand the evidence and impact of deprescribing among those living with frailty. We did not search the grey literature and may have missed some additional resources. Although we followed SWiM criteria, our synthesis of the studies should be treated with caution because of the limited number of included studies and their heterogeneity. We were also unable to perform a meta-analysis because of the heterogeneity of outcomes within the included studies.

\section{Conclusion}

This review highlights the paucity of published literature on deprescribing among older people living with frailty. The included studies used objective frailty measures and thus may not capture all studies that included frail older people. Studies were heterogenous in their settings, designs and outcomes reported making it difficult to make definite conclusions. However, we suggest that deprescribing could be safe, feasible, well tolerated and can lead to important benefits on geriatric conditions such as depression, function and frailty. Deprescribing interventions in this review appear to be effective whether led by pharmacists or multidisciplinary teams using explicit or implicit tools. This has implications for clinical practice as deprescribing could be effectively led by pharmacists in liaison with GPs in community settings, whereas multidisciplinary teams (with or without access to pharmacists) could play a key role in deprescribing in acute settings. However, more research is needed in the area of deprescribing and frailty and future studies should include those living with frailty in their samples. Moreover, in order to address the gap in our understanding of the effectiveness of deprescribing interventions on reducing and reversing frailty, or stopping its progression, adequately powered randomised controlled trials that include reliable measures of frailty should be conducted.

\section{Appendix}

Search strategy

1. exp. aged/

2. (elder" or geriatric" or older* or aged or aging or ageing).mp. [ $\mathrm{mp}=$ title, abstract, heading word, drug trade name, original title, device manufacturer, drug manufacturer, device trade name, keyword, floating subheading word, candidate term word]

3. 1 or 2

4. frailty/

5. frail".mp. [mp = title, abstract, heading word, drug trade name, original title, device manufacturer, drug manufacturer, device trade name, keyword, floating subheading word, candidate term word]

6. 4 or 5

7. 3 and 6

8. deprescription/

9. inappropriate prescribing/

10. polypharmacy/

11. (deprescrib* or deprescription* or polypharmacy or de-prescrib* or de-prescription* or poly-

pharmacy).mp. [mp = title, abstract, heading word, drug trade name, original title, device manufacturer, drug manufacturer, device trade name, keyword, floating subheading word, candidate term word]

12. 8 or 9 or 10 or 11

13. 7 and 12

\section{Abbreviations}

PRISMA: The Preferred Reporting Items for Systematic reviews and MetaAnalyses; PROSPERO: The international prospective register of systematic reviews; PIMs: Potentially Inappropriate Medications; RCTs: Randomized controlled trials; SWiM: The Synthesis Without Meta-analysis; CFS: The Canadian Clinical Frailty Score; ISAR: Identification of Seniors At Risk; e-FI: The Electronic Frailty Index; GP: General Practitioner; MERA: Medication Rationalization; MDT: Multidisciplinary team; PDP: Poly-De-Prescribing; GDS: The geriatric depression scale; MMSE: Mini Mental State Examination; QoL: Quality of life

\section{Acknowledgments}

We thank Paula Sands, Health Services librarian for her support with developing the search strategies.

\section{Authors' contributions}

$\mathrm{KI}, \mathrm{NC}, \mathrm{JMS}, \mathrm{SL}, \mathrm{SF}$ and HCR contributed to the conception and design of the review. KI \& NC completed literature search and screening, KI \& JMS extracted data from included studies and SL \& SF assessed the quality of studies. Kl drafted the manuscript and HCR contributed editing. All authors read and approved the final manuscript.

\section{Funding}

Authors did not receive funding to complete this review. However, authors receive support from the National Institute of Health Research (NIHR). H.C.R, SF, SL and K. I receive support from the NIHR Applied Research Collaboration (ARC) Wessex. KI, N.J.C and H.C.R receive support from the NIHR

Southampton Biomedical Research Centre. The views expressed are those of the authors and not necessarily those of the NHS, the NIHR, or the

Department of Health. 


\section{Availability of data and materials}

The datasets used and/or analysed during the current study are available from the corresponding author on reasonable request.

\section{Declarations}

Ethics approval and consent to participate

Not applicable.

\section{Consent for publication}

Not applicable.

\section{Competing interests}

The authors declare that they have no competing interests.

\section{Author details}

${ }^{1}$ Academic Geriatric Medicine, Faculty of Medicine, University of Southampton, Southampton, UK. ${ }^{2}$ NIHR Applied Research Collaboration Wessex, Southampton, UK. ${ }^{3}$ Southampton Biomedical Research Centre, University of Southampton and University Hospital Southampton NHS FT, Southampton, UK. ${ }^{4}$ Institute of Pharmaceutical Science, King's College London, London, UK. 'Pharmacy Department, Guy's and St. Thomas' NHS FT, London, UK. 'Primary Care, Population Science and Medical Education, Faculty of Medicine, University of Southampton, Southampton, UK.

\section{Received: 17 November 2020 Accepted: 8 April 2021}

\section{Published online: 17 April 2021}

\section{References}

1. Masnoon N, Shakib S, Kalisch-Ellett L, Caughey GE. What is polypharmacy? A systematic review of definitions. BMC Geriatr. 2017;17(1):230. https://doi. org/10.1186/s12877-017-0621-2.

2. Morley JE, Vellas B, van Kan GA, Anker SD, Bauer JM, Bernabei R, et al. Frailty consensus: a call to action. J Am Med Dir Assoc. 2013;14(6):392-7. https:// doi.org/10.1016/j.jamda.2013.03.022.

3. Gnjidic D, Hilmer SN. Use of potentially inappropriate medications in the care of frail older people. Aging Health. 2010;6(6):705-16. https://doi.org/1 0.2217/ahe.10.78.

4. Christensen M, Lundh A. Medication review in hospitalised patients to reduce morbidity and mortality. Cochrane Database Syst Rev. 2013;2. https://doi.org/10.1002/14651858.CD008986.pub2.

5. Chang C-B, Chen J-H, Wen C-J, Kuo H-K, Lu IS, Chiu L-S, et al. Potentially inappropriate medications in geriatric outpatients with polypharmacy: application of six sets of published explicit criteria. Br J Clin Pharmacol. 2011;72(3):482-9. https://doi.org/10.1111/j.1365-2125.2011.04010.x.

6. Frailty, polypharmacy and deprescribing. Drug Ther Bull. 2016;54(6):69-72.

7. Hilmer SN, McLachlan AJ, Le Couteur DG. Clinical pharmacology in the geriatric patient. Fundam Clin Pharmacol. 2007;21(3):217-30. https://doi. org/10.1111/j.1472-8206.2007.00473.x.

8. Kutner JS, Blatchford PJ, Taylor DH, Ritchie CS, Bull JH, Fairclough DL, et al. Safety and benefit of discontinuing statin therapy in the setting of advanced, life-limiting illness: a randomized clinical trial. JAMA Intern Med. 2015;175(5):691-700. https://doi.org/10.1001/jamainternmed.2015.0289.

9. Hilmer SN, Mager DE, Simonsick EM, Ling SM, Windham BG, Harris TB, et al. Drug burden index score and functional decline in older people. Am J Med. 2009;122(12):1142-9.e1-2.

10. Jyrkkä J, Enlund H, Lavikainen P, Sulkava R, Hartikainen S. Association of polypharmacy with nutritional status, functional ability and cognitive capacity over a three-year period in an elderly population. Pharmacoepidemiol Drug Saf. 2011;20(5):514-22. https://doi.org/10.1002/pds.2116.

11. Rawle MJ, Cooper R, Kuh D, Richards M. Associations between polypharmacy and cognitive and physical capability: a British birth cohort study. J Am Geriatr Soc. 2018;66(5):916-23. https://doi.org/10.1111/jgs.15317.

12. Hubbard RE, O'Mahony MS, Woodhouse KW. Medication prescribing in frail older people. Eur J Clin Pharmacol. 2013;69(3):319-26. https://doi.org/10.1 007/s00228-012-1387-2.

13. Gutiérrez-Valencia M, Izquierdo M, Cesari M, Casas-Herrero Á, Inzitari M, Martínez-Velilla N. The relationship between frailty and polypharmacy in older people: a systematic review. Br J Clin Pharmacol. 2018;84(7):1432-44. https://doi.org/10.1111/bcp.13590.
14. Stevenson JM, Davies JG, Martin FC. Medication-related harm: a geriatric syndrome. Age Ageing. 2019;49(1):7-11. https://doi.org/10.1093/ageing/afz121.

15. Veronese N, Stubbs B, Noale M, Solmi M, Pilotto A, Vaona A, et al. Polypharmacy is associated with higher frailty risk in older people: an 8-year longitudinal cohort study. J Am Med Dir Assoc. 2017;18(7):624-8. https://doi. org/10.1016/j.jamda.2017.02.009.

16. Thompson W, Farrell B. Deprescribing: what is it and what does the evidence tell us? Can J Hospital Pharmacy. 2013;66(3):201-2. https://doi. org/10.4212/cjhp.v66i3.1261.

17. Woodward MC. Deprescribing: achieving better health outcomes for older people through reducing medications. J Pharm Pract Res. 2003;33(4):323-8. https://doi.org/10.1002/jppr2003334323.

18. Curtin D, Gallagher P, O'Mahony D. Deprescribing in older people approaching end-of-life: development and validation of STOPPFrail version 2. Age Ageing. 2021;50(2):465-71. https://doi.org/10.1093/ageing/afaa159. PMID: 32997135.

19. Thompson W, Lundby C, Graabæk T, Nielsen DS, Ryg J, Søndergaard J, et al. Tools for Deprescribing in frail older persons and those with limited life expectancy: a systematic review. J Am Geriatr Soc. 2019;67(1):172-80. https://doi.org/10.1111/jgs.15616.

20. Rankin A, Cadogan CA, Patterson SM, Kerse N, Cardwell CR, Bradley MC, et al. Interventions to improve the appropriate use of polypharmacy for older people. Cochrane Database Syst Rev. 2018;9:Cd008165.

21. Iyer S, Naganathan V, McLachlan AJ, Le Couteur DG. Medication withdrawal trials in people aged 65 years and older: a systematic review. Drugs Aging. 2008;25(12):1021-31. https://doi.org/10.2165/0002512-200825120-00004.

22. Kua CH, Mak VSL, Huey Lee SW. Health outcomes of Deprescribing interventions among older residents in nursing homes: a systematic review and meta-analysis. J Am Med Dir Assoc. 2019;20(3):362-72.e11.

23. Almutairi $\mathrm{H}$, Stafford A, Etherton-Beer C, Flicker L. Optimisation of medications used in residential aged care facilities: a systematic review and meta-analysis of randomised controlled trials. BMC Geriatr. 2020;20(1):236. https://doi.org/10.1186/s12877-020-01634-4.

24. Thio SL, Nam J, van Driel ML, Dirven T, Blom JW. Effects of discontinuation of chronic medication in primary care: a systematic review of deprescribing trials. Br J Gen Pract. 2018;68(675):e663-e72. https://doi.org/10.3399/bjgp1 $8 \times 699041$.

25. Ulley J, Harrop D, Ali A, Alton S, Fowler DS. Deprescribing interventions and their impact on medication adherence in community-dwelling older adults with polypharmacy: a systematic review. BMC Geriatr. 2019;19(1):15. https:// doi.org/10.1186/s12877-019-1031-4.

26. Thillainadesan J, Gnjidic D, Green S, Hilmer SN. Impact of Deprescribing interventions in older hospitalised patients on prescribing and clinical outcomes: a systematic review of randomised trials. Drugs Aging. 2018; 35(4):303-19. https://doi.org/10.1007/s40266-018-0536-4.

27. Sanford AM, Orrell M, Tolson D, Abbatecola AM, Arai H, Bauer JM, et al. An international definition for "nursing home". J Am Med Dir Assoc. 2015;16(3): 181-4. https://doi.org/10.1016/j.jamda.2014.12.013.

28. Faller JW, DdN P, de Souza S, Nampo FK, Orlandi FS, Matumoto S. Instruments for the detection of frailty syndrome in older adults: a systematic review. PLoS One. 2019;14(4):e0216166-e.

29. Clegg A, Young J, lliffe S, Rikkert MO, Rockwood K. Frailty in elderly people. Lancet (London, England). 2013;381(9868):752-62.

30. Moher D, Liberati A, Tetzlaff J, Altman DG. Preferred reporting items for systematic reviews and meta-analyses: the PRISMA statement. BMJ. 2009; 339(jul21 1):b2535. https://doi.org/10.1136/bmj.b2535.

31. Ouzzani M, Hammady H, Fedorowicz Z, Elmagarmid A. Rayyan-a web and mobile app for systematic reviews. Syst Rev. 2016;5(1):210. https://doi.org/1 0.1186/s13643-016-0384-4.

32. R; R. Cochrane Consumers and Communication Review Group: data synthesis and analysis'. http://cccrg.cochrane.org, (Accessed 26 Nov 2019). 2013.

33. Khera S, Abbasi M, Dabravolskaj J, Sadowski CA, Yua H, Chevalier B. Appropriateness of medications in older adults living with frailty: impact of a pharmacist-led structured medication review process in primary care. J Prim Care Community Health. 2019;10:2150132719890227.

34. Ailabouni N, Mangin D, Nishtala PS. DEFEAT-polypharmacy: deprescribing anticholinergic and sedative medicines feasibility trial in residential aged care facilities. Int J Clin Pharm. 2019;41(1):167-78. https://doi.org/10.1007/s11 096-019-00784-9. 
35. Whitty R, Porter $S, R a B K$, Bhatt $P$, Koo E, et al. A pilot study of a medication rationalization (MERA) intervention. CMAJ Open. 2018;6(1):E87-94. https:// doi.org/10.9778/cmajo.20170134.

36. Curtin D, Jennings E, Daunt R, Curtin S, Randles M, Gallagher P, et al. Deprescribing in older people approaching end of life: a randomized controlled trial using STOPPFrail criteria. J Am Geriatr Soc. n/a(n/a).

37. Garfinkel D. Poly-de-prescribing to treat polypharmacy: efficacy and safety. Ther Adv Drug Safety. 2018;9(1):25-43. https://doi.org/10.1177/204209861 7736192.

38. Dalleur O, Boland B, Losseau C, Henrard S, Wouters D, Speybroeck N, et al. Reduction of potentially inappropriate medications using the STOPP criteria in frail older inpatients: a randomised controlled study. Drugs Aging. 2014; 31(4):291-8. https://doi.org/10.1007/s40266-014-0157-5.

39. Reeve E, Denig P, Hilmer SN, Ter Meulen R. The ethics of deprescribing in older adults. J Bioethical Inq. 2016;13(4):581-90. https://doi.org/10.1007/s11 673-016-9736-y.

40. Page AT, Clifford RM, Potter K, Schwartz D, Etherton-Beer CD. The feasibility and effect of deprescribing in older adults on mortality and health: a systematic review and meta-analysis. Br J Clin Pharmacol. 2016;82(3):583623. https://doi.org/10.1111/bcp.12975.

41. Garfinkel D, Mangin D. Feasibility study of a systematic approach for discontinuation of multiple medications in older adults: addressing PolypharmacyDiscontinuation of multiple dugs in older adults. JAMA Intern Med. 2010;170(18):1648-54.

42. Garfinkel D, Zur-Gil S, Ben-Israel H. The war against polypharmacy: a new cost-effective geriatric-palliative approach for improving drug therapy in disabled elderly people. Imaj Ramat Gan. 2007;9(6):430.

43. Beuscart JB, Pont LG, Thevelin S, Boland B, Dalleur O, Rutjes AWS, et al. A systematic review of the outcomes reported in trials of medication review in older patients: the need for a core outcome set. Br J Clin Pharmacol. 2017;83(5):942-52. https://doi.org/10.1111/bcp.13197.

44. Ng TP, Feng L, Nyunt MS, Feng L, Niti M, Tan BY, et al. Nutritional, physical, cognitive, and combination interventions and frailty reversal among older adults: a randomized controlled trial. Am J Med. 2015;128(11):1225-36.e1.

45. Mach J, Gemikonakli G, Logan C, Vander Wyk B, Allore H, Ekambareshwar S, et al. Chronic polypharmacy with increasing drug burden index (DBI) exacerbates frailty and impairs physical function, with effects attenuated by deprescribing, in aged mice. J Gerontol A Biol Sci Med Sci. 2020. https://doi. org/10.1093/gerona/glaa060.

46. Rolfson DB, Majumdar SR, Tsuyuki RT, Tahir A, Rockwood K. Validity and reliability of the Edmonton frail scale. Age Ageing. 2006;35(5):526-9. https:// doi.org/10.1093/ageing/afl041.

47. NCT. The impact of statin holiday in dialysis patients over 70 years old on mental function, physical function and frailty. https:/www.clinicaltrials.gov/ show/nct03663049; 2018

48. ACTRN. Opti-med: a randomised controlled trial of deprescribing to optimise health outcomes for frail older people. http://www.whoint/trialsea rch/trial2aspx?Trialid=actrn12613001204730; 2013.

49. Eckert KA, Shi Z, Taylor AW, Wittert G, Price K, Goldney RD. Learning from an epidemiological, population-based study on prescribed medicine use in adults. Pharmacoepidemiol Drug Saf. 2013;22(3):271-7. https://doi.org/10.1 002/pds.3380

50. Pruskowski JA, Springer S, Thorpe CT, Klein-Fedyshin M, Handler SM. Does deprescribing improve quality of life? A systematic review of the literature. Drugs Aging. 2019;36(12):1097-110. https://doi.org/10.1007/s40266-019-0071 7-1.

51. Lee J, Negm A, Wong E, Holbrook A. Does deprescribing fall-associated drugs reduce falls and its complications?: a systematic review. Innov Aging. 2017:1(suppl_1):268.

52. Verrue CL, Petrovic M, Mehuys E, Remon JP, Vander SR. Pharmacists' interventions for optimization of medication use in nursing homes. Drugs Aging. 2009;26(1):37-49. https://doi.org/10.2165/0002512-200926010-00003.

53. Tjia J, Velten SJ, Parsons C, Valluri S, Briesacher BA. Studies to reduce unnecessary medication use in frail older adults: a systematic review. Drugs Aging. 2013;30(5):285-307. https://doi.org/10.1007/s40266-013-0064-1.

54. Woodford HJ, Fisher J. New horizons in deprescribing for older people. Age Ageing. 2019;48(6):768-75. https://doi.org/10.1093/ageing/afz109.

\section{Publisher's Note}

Springer Nature remains neutral with regard to jurisdictional claims in published maps and institutional affiliations.

Ready to submit your research? Choose BMC and benefit from:

- fast, convenient online submission

- thorough peer review by experienced researchers in your field

- rapid publication on acceptance

- support for research data, including large and complex data types

- gold Open Access which fosters wider collaboration and increased citations

- maximum visibility for your research: over $100 \mathrm{M}$ website views per year

At BMC, research is always in progress.

Learn more biomedcentral.com/submissions 